\title{
The Qualitative Fundamental Motor Performance Characteristics of Preadolescent Obese Children
}

\author{
Steven Smith*, Kyle Morrison, Elizabeth Bransdorfer and Samuel Barthel
}

Hope College Kinesiology, Foundation for Fitness, 222 Fairbanks Ave., Holland, MI 49423, USA

\begin{abstract}
This study examined the qualitative motor performance characteristics of 35 preadolescent obese children (18 female, 17 male) in the Midwest of the United States. An available sample of children ages six to 13 were classified as obese based on a BMI score of 30 or greater. The Test of Gross Motor Development-2 (TGMD-2)was used to assess all subjects. This test measures the qualitative motor ability of children using two subtest categories of fundamental motor skills including locomotors (running, hopping, skipping, jumping, leaping and galloping) as well as object control skills (throwing, catching, kicking, bouncing a ball, rolling a ball and striking). All participants received a raw score, standardized score, sum of standards and gross motor quotient score. All scores were compared to national norms established by the authors of the TGMD-2. The results indicated that the group norms of the obese children were significantly below the mean scores of the national average for all measures including locomotor standard scores $(M=3.80, S D=2.44, p<0.001)$ and object-control standard scores $(M=4.43, S D=2.89, p<0.001)$ and the gross motor development quotient $(M=64.69, S D=15.05, p<0.001)$. The researchers concluded that the significantly lower motor performance scores of obese children may lead this population to participate less in health enhancing movement opportunities as they grow into adolescence and adulthood. The authors noted that the TGMD-2 is designed for children ages 3-10 and has a significant ceiling effect for older children. A younger population may reveal more robust conclusions in further study. Additionally, further study is recommended to determine whether programs aimed at lowering obesity levels in children can have an impact on qualitative fundamental motor skill performance.
\end{abstract}

Keywords: Kinesiology, obesity, pedagogy, Body Mass Index, BMI, assessment, health, physical education, motor performance, motor skills.

\section{INTRODUCTION}

Fundamental motor skills are the foundation to most movement that we do as human beings as we mature. These skills include such movement abilities as running, hopping, skipping and jumping (locomotor skills) as well as bouncing a ball, kicking, throwing and catching (object control skills). When these skills reach maturity, individuals can participate in the sports and games of society that are appropriate to continuing moving for a lifetime. When they are not fully developed to maturity it is clear that many individuals stop participating in those health enhancing activities such as sports, games and fitness activities. This study examined the fundamental motor performance characteristics of preadolescent obese children from a qualitative perspective. It was hypothesized that the fundamental motor performance characteristics of this population are significantly below their same-aged peers. This hypothesis was tested using the Test of Gross Motor Development-2 [1].

\section{BACKGROUND}

Obesity related co-morbidities are the number one preventable causes of death in the United States,

*Address correspondence to this author at the Hope College Kinesiology, Foundation for Fitness, 222 Fairbanks Ave. Holland, MI 49423, USA; Tel: 616-403-9550; Fax: 616-395-7195; E-mail: sdsmith@hope.edu surpassing accidents, violence, smoking, and alcohol and drug addictions. Programs that provide early identification and treatment of obesity and related comorbidities in children are critical to the future health of our community. Current information from the Center for Disease Control (CDC) indicates that $17 \%$ (or 12.7 million) of children and adolescents aged 2-19 years are obese [2].

Studies have shown that obesity significantly impacts a child's quality of life. A recent study on quality of life indicators for obese children in the Journal of the American Medical Association showed that obese children had a startlingly low quality of life. Obese children's quality of life indicators were equivalent to young cancer patients on chemotherapy. Many of the children reported the development of obesity related ailments including fatty liver disease, obstructive sleep apnea, diabetes and orthopedic problems. Obese children who reported no physical conditions still reported low quality of life [3].

Through the Holland Community Hospital's Community Outreach Department, Holland Michigan area elementary school-aged children have been screened for several years for risk factors associated with Type 2 Diabetes. Obesity is a key risk factor for Type 2 Diabetes. Once found only in adults, Type 2 Diabetes is now being diagnosed at an alarming rate among children as young as 10 and 12 years of age. 
The long-term health outcomes for children who develop Type 2 Diabetes are expected to be similar to those of adults. Health impacts include fetal loss and malformation in pregnancy, blindness, kidney failure, myocardial infarction, stroke, amputation of feet, nerve disease, depression, tooth loss and gum disease, and premature death. Unfortunately, rather than exhibiting these health complications at 60 or 65 , children who develop Type 2 Diabetes in their early teens can expect the onset of complications in their late 20 s or 30s.

Recently, the Holland Community Hospital's Community Outreach Department found that $83 \%$ of the fourth graders screened had at least one risk factor associated with Type 2 Diabetes. With a student population which is $43 \%$ Hispanic, many of our community's children are at risk based on ethnicity. In addition, $41 \%$ of the students screened had Body Mass Indexes (BMI) at the $85^{\text {th }}$ percentile or greater (at-risk for obesity.) Twenty percent of the students had BMl's at the $95^{\text {th }}$ percentile or greater (obese). These values are $10 \%$ higher than the State of Michigan average and Michigan is ranked among the five fattest states [4].

This study focused on discovering if the qualitative motor performance characteristics of obese children may be having an impact on their movement abilities. Fundamental motor skills are essential in performing most of the physical activities that children participate in on playgrounds and in the sport arena as they grow older. If a child is unable to reach maturity in these basic skills it is likely that they will run into a proficiency barrier and avoid participation in movement later in life. This pattern can reinforce the cycle that leads to continued obesity.

The goal of this project was to identify the qualitative fundamental motor skill characteristics of obese children and compare them to national norms. The faculty and student researchers worked together to complete the data collection in the context of a Foundation for Fitness program at Hope College in Holland, Michigan. The lead investigator trained the student researchers in the use of the TGMD-2 instrument.

\section{METHODS}

\section{Subjects}

The subjects for this study ranged in age from 6-13 years (see Table 1) and were acquired through an available sample from individuals who were enrolled in the Foundation for Fitness (FFF) program at Hope College. These children were identified by the school health system or by area physicians as students who could benefit from a program of intervention due to their measured obesity. Obesity for this study was defined by the standard characteristics of a body mass index (BMI) of 30 or greater (a standard measure for defining obesity). The program also screened for other factors according to the standards of the FFF (not including students with other metabolic disorders or disabling conditions). In order to meet the needed sample size we sought referrals from Holland Pediatrics and the school nurses program of area public schools. Human subjects review was accomplished for all stages of the FFF program.

\section{Assessment and Instrumentation}

Motor skills develop in a clear and identifiable sequence and these sequences have been quantified in the Test of Gross Motor Development (TGMD-2) instrument. This test examines the presence or absence of mature fundamental motor skills such as running, hopping, skipping, and bouncing a ball, throwing, catching and other similar skills. Unique to this qualitative test is rather than examining how far and how fast a student performs (often typical in physical education), this instrument uses a criterionreferenced approach to measure the appearance and maturity of the performer while attempting the skill. An example of throwing from the manual is described as:

\section{THROWING:}

- Windup is initiated with downward movement of the hand/arm

- $\quad$ Rotates hip and shoulders to a point where the non-throwing side faces the wall

Table 1:

\begin{tabular}{|c|c|c|c|c|c|}
\hline $\mathbf{N}$ & Minimum & Maximum & Range & Mean & SD \\
\hline $\begin{array}{c}35 \\
F=51 \% \\
M=49 \%\end{array}$ & 6.22 & 13.11 & 6.89 & 9.98 & 1.737 \\
\hline
\end{tabular}


- Weight is transferred by stepping with the foot opposite the throwing hand

- Follow-through beyond ball release diagonally across the body toward the non-preferred side

Though this test is qualitative in it's approach, the test produces a quantifiable score allowing for national norm comparison of groups. The validity and reliability of this instrument is widely established by the test developers.

The researchers for this study collected data of children performing the various skills and establishing inter-rater reliability. Agreement of the lead researcher and student researchers of the presence or absence of the mature characteristics of the skill listed on the test met a $98 \%$ agreement rate. The TGMD-2 produces a locomotor subtest and an object control subtest. Each portion of the test involves multiple skills in their respective categories. The researchers were taught the criterion to be referenced and used film to analyze children performing the skill. The researchers analyzed the data including raw scores, percentile rankings, standard scores, sum of standard scores, and gross motor development quotient, age equivalents, and zscores.

Each child performed 12 fundamental motor skills at various stations in a gymnasium setting. The stations were filmed in order to increase reliability of the analyzers of the skills. The presence or absence of the mature characteristics of the skill was measured during two trials of each of the skills. Totals of the trial scores for each skill were attained and then further conversion of scores took place for statistical analysis (adjusted by age for comparisons across ages).

\section{RESULTS}

The results of the data indicated significant differences between the study participants and the national norms developed by the TGMD-2. T-test comparisons revealed that the children in the FFF program with obesity scored significantly lower than the national norms on measures of qualitative motor performance. The TGMD-2 allows for comparisons on the locomotor subscale standard scores, the object-

Table 2:

\begin{tabular}{|c|c|c|c|c|c|}
\hline & & Mean & $\mathbf{N}$ & Std. Deviation & Std. Error Mean \\
\hline \multirow[t]{2}{*}{ Pair 1} & Standard Loc & 3.800 & 35 & 2.4471 & .4136 \\
\hline & NAT LOC STANDARD MEDIAN & 10.000 & 35 & .0000 & .0000 \\
\hline \multirow[t]{2}{*}{ Pair 2} & Standard OC & 4.429 & 35 & 2.8931 & .4890 \\
\hline & NAT OC STANDARD MEDIAN & 10.000 & 35 & .0000 & .0000 \\
\hline \multirow[t]{2}{*}{ Pair 3} & GMDQ & 64.686 & 35 & 15.0544 & 2.5447 \\
\hline & NAT GMDQ & 100.000 & 35 & .0000 & .0000 \\
\hline \multirow[t]{2}{*}{ Pair 4} & Percentile & 6.286 & 35 & 13.1162 & 2.2170 \\
\hline & NAT PERCENTILE & 50.000 & 35 & .0000 & .0000 \\
\hline
\end{tabular}

Table 3:

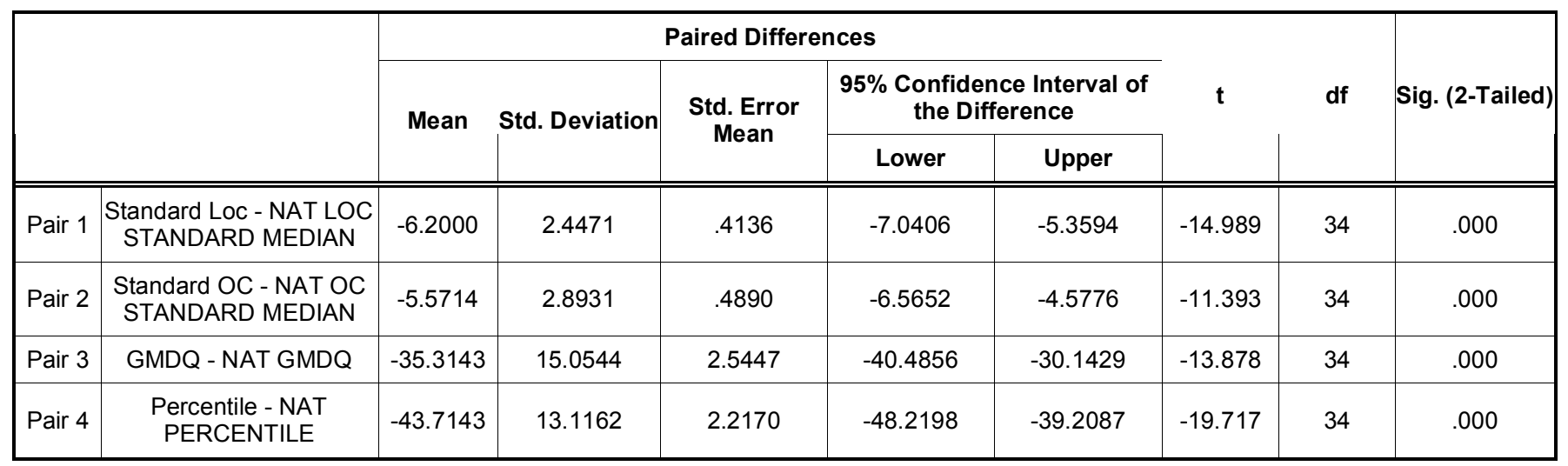


control subscale standard scores, a combined gross motor development quotient and percentile rankings. A separate analysis allows for age equivalency to be determined for each individual as well.

The locomotor standard scores revealed that the obese children in this study had significantly lower $(p<0.001)$ mean standard scores of 3.8 while national norm scores are at a mean of 10 . The object control mean scores showed similar results with significantly lower mean scores $(p<0.001)$ of 4.4 compared to national norm scores of 10 (see Tables 2 and 3 ).

\section{DISCUSSION}

It is clear that for the children tested there is a significant lag in motor skill ability when compared to national norms of same aged peers. What is not known is whether the children are lagging in ability due to their obesity and whether the lag in motor skill ability is causative in their obesity levels.

It is important for physical educators and others who hope to intervene in the obesity crisis to try to determine whether poor motor skills are a contributing factor to obesity. It is possible that children who have poor motor skill ability simply choose not to participate in the games and sports of our culture because of the lack of ability. Conversely, it is also possible that the obesity itself causes children to not be able to function well in qualitative motor performance. For example, the ability for obese children to propel themselves up into the air for such tasks as jumping, hopping, skipping and sliding is very limited due to the strength it would take to move the extra weight. The inability to do so may cause the child to continue to avoid such activities leading to even less physical activity and the games and sports that would require such activities. It is evident that children need to spend significant time on each stage of development to reach maturity in each of the fundamental motor skills tested on the TGMD-2. The younger a child exhibits characteristics of obesity the more likely that they will not reach maturity in the skills if they start to avoid the physical activity.

We are also aware that principles of motor development inform us that there is a period of readiness when these skills can be most efficiently learned. Some would argue that unless they are learned during a very plastic time of their neurological development they will simply never reach their full potential or maturity. In a recent study by Morrison [5], it is reported that for basic fundamental motor skill development the period of readiness is likely in sync with the neural plasticity of the brain development which is limited after age eight with $90 \%$ of development already attained [6].

Morrison also states that the interaction of motor performance with perceived competence is an important variable when choosing to participate in physical activity [5]. He states that perceived athletic competence explains far greater variance in childhood physical activity than does motor performance. This may mean that the psychological factors of perceived ability may lead poor performers in fundamental motor skills to avoid physical activity simply based on their perceptions of their abilities.

Seefeldt and Haubenstricker [7] also proposed in their well-designed model of motor development the concept of a proficiency barrier. In their model of the progression of motor development they recognize that motor skills build on the foundation of environmental and maturational influences. The skills that we attain in our lifespan begin with reflexes, then progress to fundamental motor skills (the ones tested in the TGMD2 ). They then place a proficiency barrier above fundamental motor skills to represent the concept that unless children become proficient in the lower levels of the model they will never attain the higher levels of the model that include transitional skills and the sports, games and dances of the culture.

The findings of this study are important when we consider the model described above. If we desire to see all children (including currently obese children) to be movers for a lifetime and avoid the high risks of a sedentary lifestyle it can be very important for children to develop these fundamental motor skills. These researchers believe that if these skills are not reaching maturity by the younger elementary years of ages three to eight it is likely that maturity will never be reached and avoidance of physical activity requiring these skills will be inevitable.

\section{ACKNOWLEDGEMENTS}

The authors would like to thank the Herman Miller Foundation for funding the grant that supports the Foundation for Fitness program at Hope College. Additionally we would like to thank the Provost Grant for Student-Faculty Collaborative Research at Hope College for funding the student researchers.

\section{REFERENCES}

[1] Ulrich, DA, The test of gross motor development, TGMD-2. Austin, Texas: Pro-Ed 2000. 
[2] Center for Disease Control Website, [cited 2015 Nov 22] Available from: http://www.cdc.gov/obesity/datal childhood.html

[3] Center for Disease Control Website, [cited 2015 Nov 22] Available from: http://www.cdc.gov/obesity/data/prevalencemaps.html.

[4] Schwimmer JB, Burwinkle TM, Varni JW. Health related quality of life of severely obese children and adolescents. JAMA 2003; 289: 1813-9.

http://dx.doi.org/10.1001/jama.289.14.1813
[5] Morrison KM. The inter-relationships among physical activity, motor performance and perceived athletic competence in normal and overweight/obese children (Doctoral dissertation) 2015. Retrieved from Proquest.

[6] Scammon RE. The measurement of the body in childhood. In The measurement of man, edited by J.A. Harris et al. (Minneapolis, MN: University of Minnesota Press) 1930; 193.

[7] Seefeldt V, Haubenstricker J. Developmental Sequences of Fundamental Motor Skills, Unpublished research, Michigan State University 1976.

http://dx.doi.org/10.6000/1929-4247.2016.05.04.2 